\title{
Ribociclib as New Cause of Cornea Verticillata
}

\author{
Alba Linero Carmen ${ }^{1,2 *}$, Rocha de Lossada Carlos ${ }^{1}$, Rodríguez Calvo de Mora Marina ${ }^{1}$, De las Rivas Ramírez Nieves ${ }^{1}$, \\ Espejo de los $\operatorname{Riscos}^{1}$ and Alba Emilio ${ }^{3,4}$
}

${ }^{1}$ Department Ophthalmology, Hospital Civil of Málaga, Spain

${ }^{2}$ Department of Ophthalmology, Faculty of Medicine of Málaga, University of Málaga, Spain

${ }^{3}$ Unity of Clinical Management Intercenter of Oncology, Biomedical Research Institute of Malaga (IBIMA), Regional University Hospitals and Virgen de la Victoria, Spain

${ }^{4}$ Laboratory of Molecular Biology of Cancer, Medical-Health Research Center (CIMES), University of Málaga, Spain.

*Corresponding author: Alba-Linero Carmen, Department Ophthalmology, Faculty of Medicine of Málaga, University of Málaga,

Hospital Civil of Málaga, Spain

\begin{abstract}
Our purpose is to describe the appearance of a new origin of Cornea Verticillata through a clinical case of a 67-years-old female patient with breast cancer who was being treated by Ribociclib and attended the consultation of ophthalmology by halos vision. Exploration was performed by visual acuity, slit lamp examination, fluorescein staining, retinography and corneal topography. We observed cornea verticillata in our patient, she did not need specific treatment and the findings remained stable over time.
\end{abstract}

Keywords: Cornea Verticillata; Ribociclib; Breast cancer; Adverse effect; Amiodarone

\section{Introduction}

Cornea Verticillata a is an often asymptomatic and reversible corneal alteration caused by certain diseases and drugs $[1,2]$. Our goal is to announce a new cause of this finding. Ribociclib is a

\section{Clinical Case}

Figure 1: Slit-lamp image of pigmented corneal Verticillata.

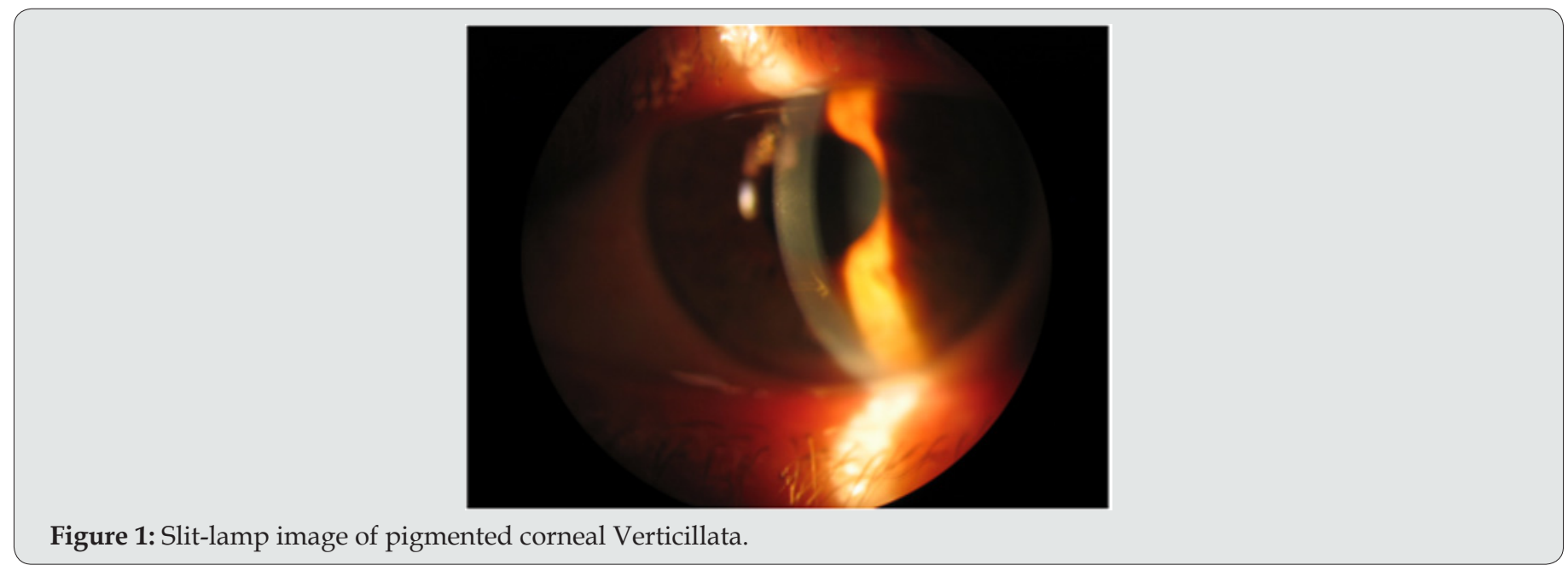

drug used in breast cancer therapy [3]. It is important to know the adverse effects of new drugs and their ocular repercussion. Herein we present a clinical case of Cornea Verticillata as a consequence of Ribociclib therapy. 
A 67-year-old woman with a history of breast cancer comes to our office for feeling halos and blurred vision. The patient was diagnosed with metastatic breast cancer for 2 years, treated by tumerectomy and oral Ribociclib (500 mg daily). She had no known ocular history or allergies. The visual acuity with its optical correction was 0.8 (decimal scale) for both eyes. In the anterior pole biomicroscopy, a transparent cornea with pigmented verticillata keratopathy was seen (Figure 1). An incipient nuclear cataract could also be observed. The rest of the parameters corresponded to normality. The intraocular pressure was $15 \mathrm{mmHG}$ in both eyes and the fundoscopy showed no significant findings. A Schirmer type I test was performed, which was $20 \mathrm{~mm}$ in the right eye and $15 \mathrm{~mm}$ in the left eye. Fluorescein staining showed no epithelial damage
(Figure 2). The corneal tomography (Pentacam, Okulus Optikgerate $\mathrm{GmbH}$, Wetzlar, Germany) revealed a regular astigmatism with the rule, with central pachymetry of 540 microns in the right eye and 530 microns in the left eye. There were no corneal thinning points or irregularities in either eye (Figure 3). Retinography and autofluorescence, as well as optical coherence tomography, did not show significant findings. Given the good visual acuity of the patient and the benign finding of verticillata cornea secondary to Ribociclib, observation and monitoring of the lesion was decided. The patient was evaluated at 3 and 6 months after the consultation, resulting in a similar exploration to the previous ones. She is currently in treatment with Ribociclib and topical lubricants on demand.

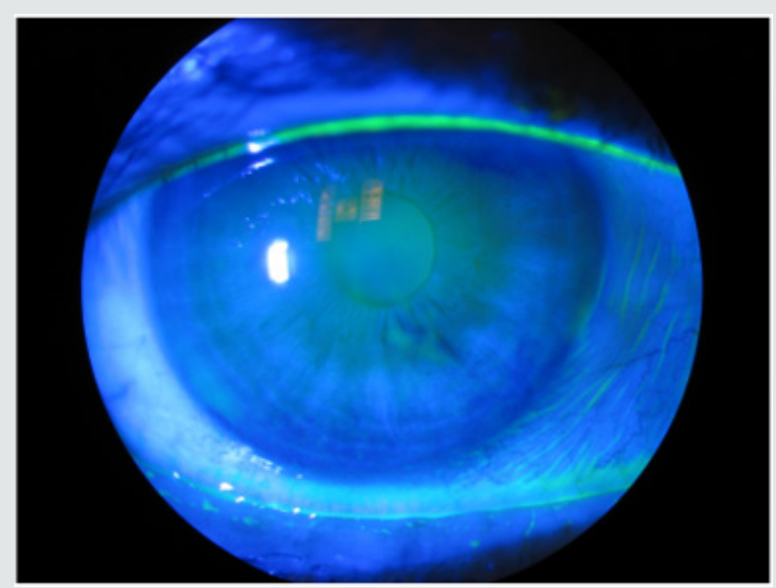

Figure 2: Fluorescein staining image showing no epithelial defect in a patient with Cornea Verticillata.

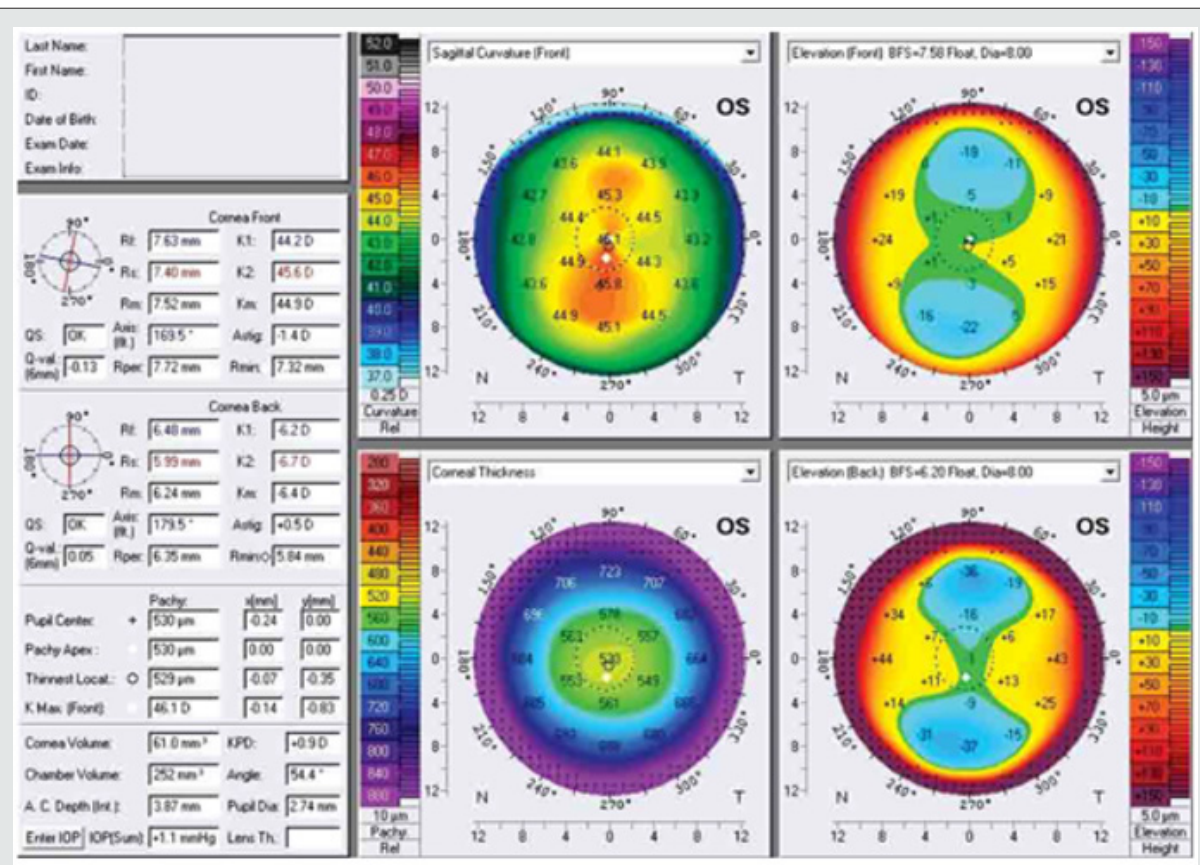

Figure 3: Corneal tomography revealing regular astigmatism with the rule and normal thickness.

\section{Discussion}

The Verticillata cornea is a keratopathy, often unnoticed, that occurs in the corneal apex after the use of certain drugs or in the context of a systemic disease [4]. Its characteristic shape allows diagnosis through biomicroscopic exploration. It is usually a benign and not very symptomatic disorder that disappears if it is secondary to a triggering factor. Among the diseases most related to this picture are Fabry disease or Cystinosis [5]. The drugs that most often cause cornea Verticillata are amiodarone, chloroquines, tamoxifen and indomethacin, among many others. Ribociclib is 
an antineoplastic drug that inhibits cyclins and is administered orally as a treatment for metastatic breast cancer [6]. Its indication is approved for breast neoplasms with positive Hormone Receptors and negative Epidermoid Growth Receptor [7]. Since the introduction of the drug, the resistance to previous therapies in this type of tumors has been reduced by $20 \%$. Among the most frequent side effects are neutropenia, alteration of liver enzymes and digestive symptoms such as nausea, diarrhea or vomiting. At present, the use of this drug has not been linked to the appearance of Verticillate cornea. It is always important to perform a detailed ocular exploration of the cornea verticillate secondary to drugs, since we can also find associated retinal toxicity. The retinography and the autofluorescence image can help us in the screening. The withdrawal of the drug is usually not necessary due to its benignity, although close monitoring of the lesions is recommended.

\section{Conclusion}

The multidisciplinary work between oncologists and ophthalmologists is necessary for the management of these patients. Ribociclib could result a new cause of Cornea Verticillate.

\section{References}

1. Reasor MJ, Hastings KL, Ulrich RG (2006) Drug-induced phospholipidosis: Issues and future directions. Expert Opin Drug Saf 5(4): 567-583.

2. Ghosh M, McCulloch C (1984) Amiodarone-induced ultrastructural changes inhuman eyes. Can J Ophthalmol 19(4): 178-186.

3. Hortobagyi GN, Stemmer SM, Burris HA, Yoon Sim Yap, Gabe S Sonke, et al. (2016) Ribociclib as firstline therapy for HR-positive, advanced breast cancer. N Engl J Med 375(18):1738-1748.

4. Ingram DV, Jaggarao NS, Chamberlain DA (1982) Ocular changes resulting from therapy with amiodarone. Br J Ophthalmol 66(10): 676679.

5. Reasor MJ, Hastings KL, Ulrich RG (2006) Drug-induced phospholipidosis: Issues and future directions. Expert Opin Drug Saf 5(4): 567-583.

6. Boér K (2017) Fulvestrant in advanced breast cancer: Evidence to date and place in therapy. Ther Adv Med Oncol 9(7): 465-479.

7. Brufsky AM (2015) Delaying chemotherapy in the treatment of hormone receptor-positive, human epidermal growth factor receptor 2-negative advanced breast cancer. Clin Med Insights Oncol 9: 137-147.
To Submit Your Article Click Here: This work is licensed under Creative Commons Attribution 4.0 License

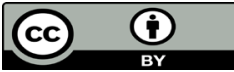

:

DOI: $10.32474 /$ TOOAJ.2019.02.000138

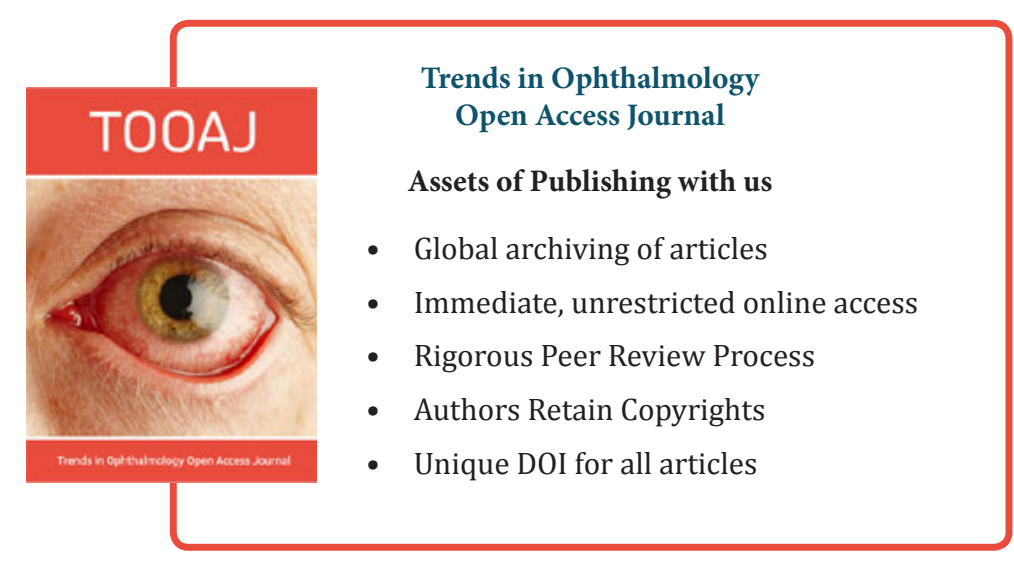

